\title{
REPOSIÇÃO AUTOMÁTICA DE CORPOS MOEDORES VIA CONTROLE AVANÇADO*
}

\section{Resumo}

Hugo Montalvão Gontijo' Marcelo Montalvão Gontijo ${ }^{2}$ Roberto Panzera ${ }^{3}$ Paula Moreira Mol Pereira ${ }^{4}$ Jonathan Felipe de Lima Silva ${ }^{5}$ Anderson Belo Fernandes ${ }^{6}$ Alex $\mathrm{Tadeu}^{7}$

O complexo de extração, beneficiamento e transporte de minério de ferro denominado Minas-Rio, de propriedade da Anglo American, consome na moagem primária uma grande quantidade de corpos moedores (bolas metálicas). Recentemente, foi desenvolvido em 4 etapas um trabalho para automatizar a reposição dos corpos moedores e estabilizar o grau de enchimento do moinho. $\mathrm{Na}$ etapa $A$, com a reposição sendo realizada com auxílio de um operador. $\mathrm{Na}$ etapa $\mathrm{B}$, com a reposição sendo realizada automaticamente de acordo com uma taxa de reposição predefinida. Nas etapas $C$ e $D$, com a taxa de reposição sendo alterada por meio do sistema de controle avançado OCS-4DC, considerando a potência do moinho como indicação indireta do grau de enchimento. É possível verificar após a implementação das modificações uma maior estabilidade da potência e consequentemente maior estabilidade do grau de enchimento.

Palavras-chave: Corpos moedores; Reposição automática; Controle avançado de processos; Moinho de bolas.

\section{AUTOMATED GRINDING MEDIA CHARGING SYSTEM USING ADVANCED PROCESS CONTROL}

\section{Abstract}

Minas-Rio, owned by Anglo American, is an iron ore extraction, processing and transportation complex that consumes a large amount of grinding media in primary grinding. Recently, a work was developed in 4 stages to automate grinding media charging and stabilize charge level of mill. In stage A, the charging was performed with supervision of an operator. In stage B, the charging was performed automatically according to a pre-set charging rate. In stages $C$ and $D$, the charging rate was manipulated by the advanced process control OCS-4D@, considering the mill power as an indirect indication of charge level. After the implementation, it is possible to verify a greater stability of the power and consequently greater stability of the charge level.

Keywords: Grinding media; automated charging; Advanced process control; Ball mill.

1 Engenheiro de Controle e Automação, Mestre, Engenheiro de Controle Avançado de Processos, Process Optimization, Metso Partners, Belo Horizonte, Minas Gerais, Brasil.

2 Engenheiro de Controle e Automação, Mestre, Engenheiro de Controle Avançado de Processos, Process Optimization, Metso Partners, Belo Horizonte, Minas Gerais, Brasil.

3 Engenheiro de Minas, Engenheiro de Vendas, Metso, Belo Horizonte, Minas Gerais, Brasil.

4 Engenheira de Produção, Engenheira de Processos, Gerência de Processo e Qualidade, Anglo American, Conceição do Mato Dentro, Minas Gerais, Brasil.

5 Engenheiro de Minas, Engenheiro de Processos, Gerência de Operação de Usina, Anglo American, Conceição do Mato Dentro, Minas Gerais, Brasil.

6 Engenheiro de Controle e Automação, Engenheiro de Automação, Gerência de Automação e Energia, Anglo American, Conceição do Mato Dentro, Minas Gerais, Brasil.

7 Engenheiro de Minas, Chefe de Vendas, Metso, Belo Horizonte, Minas Gerais, Brasil. 


\section{INTRODUÇÃO}

O projeto Minas-Rio pertencente a Anglo American está localizado nos estados de Minas Gerais e Rio Janeiro. A mina e a usina estão localizadas na cidade de Conceição do Mato Dentro em Minas Gerais, sendo esta interligada por meio de um mineroduto com $529 \mathrm{~km}$ de extensão ao porto localizado em São João da Barra no Rio de Janeiro. O complexo para extração, beneficiamento e transporte de minério de ferro engloba mina, usina, mineroduto, filtragem, estocagem e porto, sendo projetado para produzir 24,5 milhões de tonelada por ano (base seca) de Pellet Feed [1]. A Figura 1 ilustra o fluxograma com as instalações do Minas-Rio.

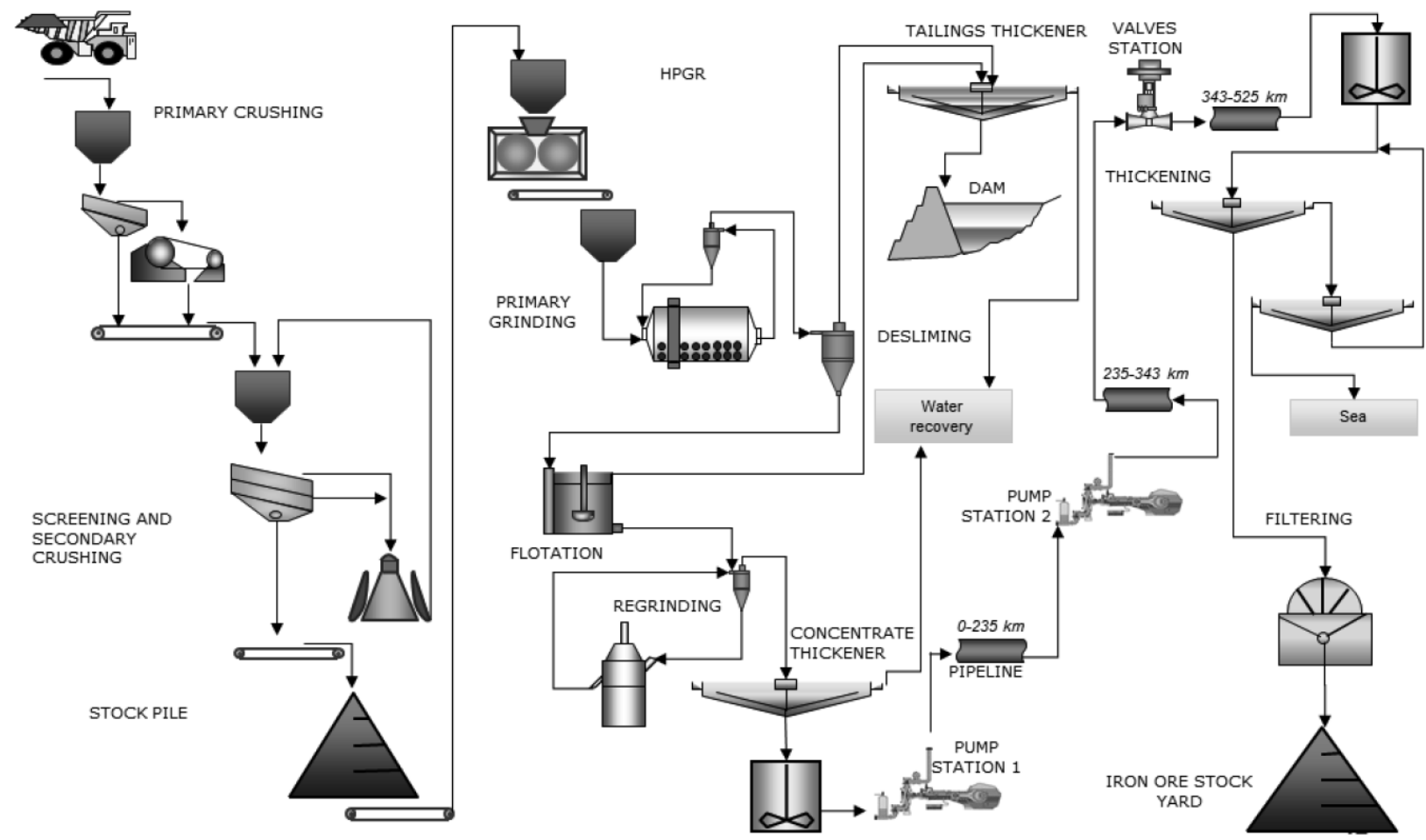

Figura 1. Fluxograma com as instalações do complexo Minas-Rio [1].

A moagem primária é composta por dois circuitos de moagem do tipo direta em paralelo. Cada circuito possui um moinho de bolas de $7,8 \mathrm{~m}$ de diâmetro por 12,6m de comprimento com 2 motores de $7500 \mathrm{~kW}$ (totalizando $15000 \mathrm{~kW}$ por circuito) e duas baterias de 8 hidrociclones de $800 \mathrm{~mm}$ de diâmetro [2].

O processo de cominuição dentro dos moinhos é realizado por corpos moedores, sendo utilizado bolas metálicas na Anglo American. Devido a diversos fatores, tais como abrasão e corrosão, as bolas metálicas desgastam com o decorrer do tempo até atingirem um diâmetro mínimo na qual são comumente expurgadas pelo moinho, sendo gastos por ano mais de 10000 toneladas de bolas nos circuitos de moagem primária.

A porcentagem do volume interno do moinho ocupado pelos corpos moedores é denominada grau de enchimento, sendo este determinado pela equipe de processo e operação de acordo com as características de alimentação e produto dos moinhos. Operacionalmente, deseja-se manter o grau de enchimento estável ao longo do 
tempo. A reposição frequente dos corpos moedores também é importante para manutenção do colar (distribuição de tamanho das bolas) ideal da carga.

Dessa forma foram realizadas pela equipe da Anglo American várias melhorias objetivando estabilizar o grau de enchimento dos moinhos. Na etapa A, foi instalado e comissionado um equipamento para pesar e repor pequenas bateladas de bolas. $\mathrm{Na}$ etapa $\mathrm{B}$, foi desenvolvida uma lógica no PLC para repor automaticamente uma determinada massa de bolas de acordo com a quantidade de horas de funcionamento dos moinhos. Nas etapas C e D, foram desenvolvidas lógicas no sistema OCS-4D(C) para variar a taxa de reposição de bolas para manter o grau de enchimento constante.

O sistema OCS-4DC) é um software de controle avançado (APC - Advanced Process Control) composto por vários módulos, modelos fenomenológicos, controle preditivo baseado em modelos (MPC - Model Predictive Control), estatísticas, interface gráfica, visão computacional e sistema especialista com lógica fuzzy. Este sistema é amplamente utilizado nas diversas etapas do beneficiamento do minério, como britagem [3], moagem [4], deslamagem [5], flotação [6] e outras. O software é customizado de acordo com o processo a ser controlado e os dados disponíveis (instrumentação e análises de laboratório). Dessa forma, quando em operação, o OCS-4DC constantemente acessa os dados de processo e laboratório, calcula e envia setpoints otimizados, conforme ilustrado na Figura 2.

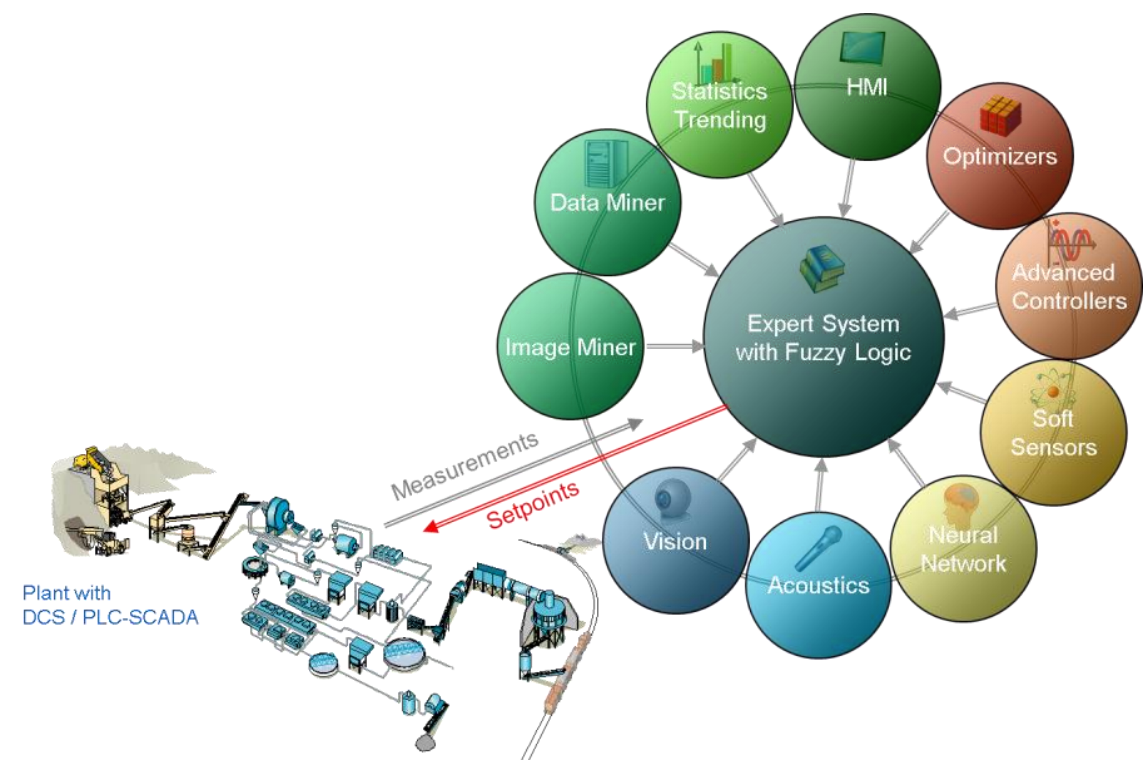

Figura 2. Otimização dos setpoints com o software OCS-4DC.

\section{MATERIAIS E MÉTODOS}

A reposição dos corpos moedores é normalmente calculada de acordo com a Equação 1:

$$
\text { Reposicao }=\frac{\text { Cons.Esp. } x \text { Pot.Cons. }}{1000}
$$

Sendo Reposição a quantidade corpo moedor desgastados que deve ser reposto em kg; Cons. Esp. o consumo específico do corpo moedor em $\mathrm{g} / \mathrm{kWh}$ e Pot. Cons. a potência consumida pelo moinho em um determinado intervalo de tempo em kWh. 
$\mathrm{Na}$ etapa $\mathrm{A}$, a reposição era realizada manualmente por um operador no campo, normalmente com frequência diária. A quantidade da total reposição era calculada pela equação 1 considerando a potência consumida desde da última reposição. No campo, o operador utilizava uma IHM existente ao lado do silo de bolas para determinar e visualizar a massa de cada batelada de reposição de bolas. Como a massa de cada batelada era limitada pelo volume do silo de pesagem (em torno de $500 \mathrm{~kg}$ ), eram necessárias realizar várias bateladas. A cada 3 meses, durante a parada para manutenção, era realizado uma medição do grau de enchimento do moinho. Se necessário, era realizado posteriormente à medição um cálculo para correção do grau de enchimento. Nessa etapa era comum ocorrer durante a reposição obstrução dos silos de bolas e do desviador existente na entrada dos moinhos. Após a realização de melhorias mecânicas e a instalação de novos sensores foi possível prosseguir para próxima etapa.

$\mathrm{Na}$ etapa $\mathrm{B}$, a reposição passou a ser realizada de forma automática. A partir de uma taxa de reposição (em $\mathrm{kg} / \mathrm{h}$ ) digitada pelo operador no supervisório uma lógica foi desenvolvida no PLC para realizar a reposição. Resumidamente, quando o moinho está em funcionamento é incrementado o valor do "totalizador de adição de bolas necessária" de acordo com o valor definido pelo operador. Sempre que possível é realizado a adição de bolas até esse totalizador zerar. Nessa etapa, ao invés de repor grande quantidade de bolas apenas uma vez por dia, passou-se a repor bolas frequentemente com bateladas menores, mas a reposição considera apenas o tempo de funcionamento sem levar em consideração a potência.

$\mathrm{Na}$ etapa $\mathrm{C}$ a taxa de reposição passou a ser determinada pelo OCS-4DC com o objetivo de estabilizar o grau de enchimento do moinho. A densidade aparente da carga está diretamente relacionada a potência do moinho conforme demonstrado na Equação 2 [2; 7]:

$$
P_{\text {liq }}=\eta P_{\text {inst }}=0,238 D^{3,5}\left(\frac{L}{D}\right) N_{c} \rho_{a p}\left(J-1,065 J^{2}\right) \operatorname{sen} \alpha
$$

Sendo $\boldsymbol{P}_{\text {liq }}$ a potência líquida em $\mathrm{kW} ; \boldsymbol{P}_{\text {inst }}$ a potência instalada em kW; $\boldsymbol{\eta}$ a eficiência de transmissão elétrica e de potência em \%; $\boldsymbol{D}$ o diâmetro interno do moinho em pés; $\boldsymbol{L}$ o comprimento efetivo do moinho em pés; $\boldsymbol{N}_{\boldsymbol{c}}$ a fração da velocidade crítica do moinho; $\boldsymbol{\rho}_{\boldsymbol{a} \boldsymbol{p}}$ a densidade aparente da carga em $\mathrm{t} / \mathrm{m}^{3} ; \boldsymbol{J} 0$ enchimento volumétrico em \% e $\boldsymbol{\alpha}$ o ângulo de levantamento da carga.

A densidade aparente da carga pode ser estimada conforme a Equação 3 [2; 8]:

$$
\rho_{a p}=\frac{\left(1-f_{v}\right) \rho_{b} J_{b}+\rho_{p} J_{p} f_{v} J_{b}+\rho_{p}\left(J-J_{b}\right)}{J}
$$

Sendo $\boldsymbol{f}_{v}$ o volume em \% de vazios intersticiais entre as bolas (tipicamente assume ser $40 \%$ do volume aparente ocupado pelas bolas); $\boldsymbol{J}_{\boldsymbol{b}}$ o enchimento aparente de bolas em \% (incluindo bolas, polpa e vazios intersticiais entre as bolas); $\boldsymbol{J}_{\boldsymbol{p}} 0$ enchimento intersticial da polpa em \% (corresponde à fração intersticial de vazios disponíveis entre a carga de bolas, ocupada pela polpa e partículas finas); $\boldsymbol{\rho}_{\boldsymbol{b}}$ o peso específico das bolas em $\mathrm{t} / \mathrm{m}^{3}$ e $\boldsymbol{\rho}_{\boldsymbol{p}}$ o peso específico da polpa em $\mathrm{t} / \mathrm{m}^{3}$. 
As Equações 2 e 3 sugerem que é possível utilizar a potência do moinho (em operação estável) como uma indicação indireta do grau de enchimento, supondo que os demais parâmetros não alteram significativamente ao longo do tempo. Dessa forma criou-se uma lógica no sistema OCS-4DC, para alterar a taxa de reposição de bolas e manter a potência em um valor desejado, definido pela operação de acordo com o grau de enchimento desejado. O sistema considera valores médios da potência apenas quando o circuito está em operação estável, desprezando os momentos de partida do circuito. A taxa de reposição é calculada de acordo com a Equação 4:

$$
\text { Taxa de reposicao }=\frac{\text { Con.Esp. } x \text { Pot. Media }}{1000}+\Delta f u z z y(4)
$$

Sendo Taxa de reposição a taxa de reposição de bolas por tempo de funcionamento do moinho em kg/h; Cons. Esp. o consumo específico do corpo moedor em g/kWh; Pot. Media a potência média do moinho em operação estável em kW e $\boldsymbol{\Delta} \boldsymbol{f} u \boldsymbol{z} \boldsymbol{y}$ uma correção da taxa de reposição em kg/h.

O primeiro termo da Equação 4 calcula a reposição "teórica" de acordo com o consumo específico e a potência média do moinho. Entretanto o desgaste real do das bolas pode ser superior ou inferior ao teórico devido a inúmeros motivos, sendo importante adicionar um fator de correção. Essa correção é calculada pelo OCS-4D utilizando lógica fuzzy e os valores de potência desejada e a potência média atual.

$\mathrm{Na}$ etapa $\mathrm{D}$, foi inserida uma correção para reduzir a influência da densidade da polpa (porcentagem de sólidos) dentro do moinho. A porcentagem de sólidos é calculada pelo OCS-4DC realizando o balanço de massas da moagem utilizando as medidas disponíveis: porcentagem de sólidos no underflow, taxa de alimentação nova, umidade da alimentação nova, densidade da polpa de alimentação dos ciclones, vazão de polpa na alimentação dos ciclones, vazão de água na entrada do moinho. Apenas a porcentagem de sólidos do underflow e a umidade de alimentação não são medidas online. Com o circuito em operação estável, variou-se a porcentagem de sólidos para obter o gráfico ilustrado na Figura 3 e 4.

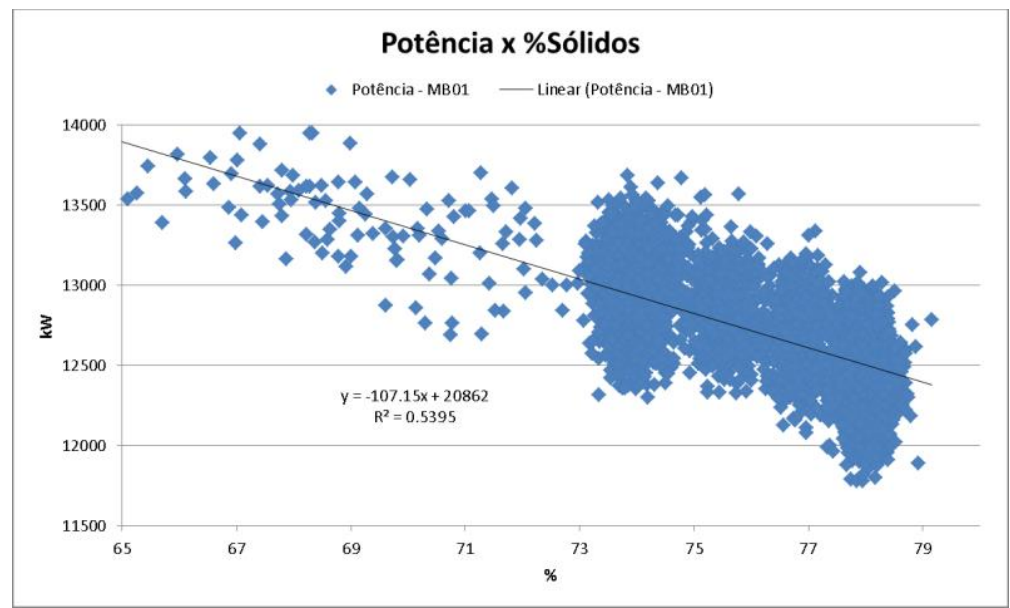

Figura 3. Potência medida versus porcentagem de sólidos do moinho (circuito 1). 


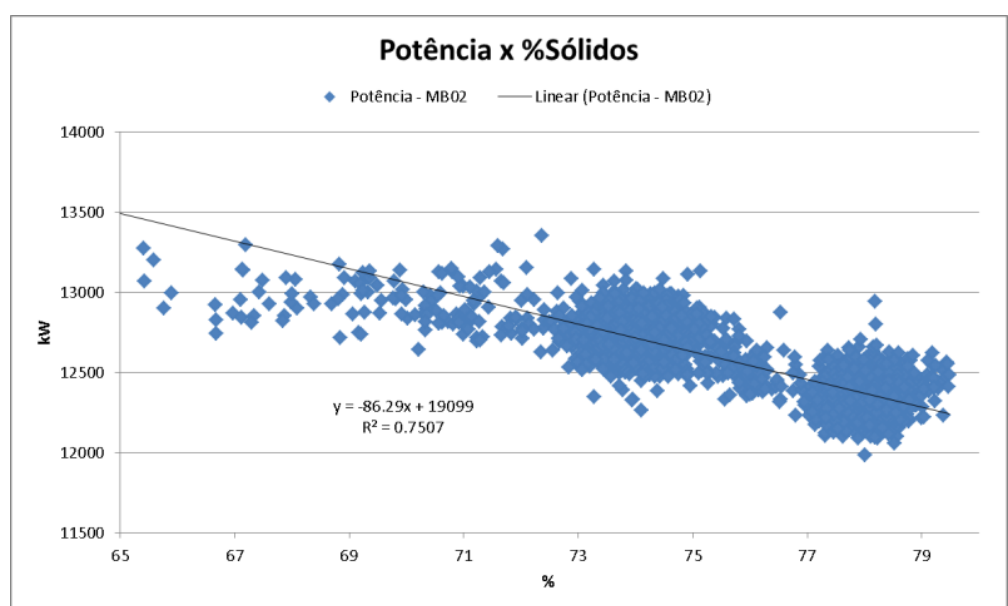

Figura 4. Potência medida versus porcentagem de sólidos do moinho (circuito 2).

Definiu-se que o valor de potência desejada configurada dentro do OCS-4DC deve ser sempre definida considerando-se uma porcentagem sólidos do moinho de $76 \%$. A partir dos dados ilustrados nas Figuras 3 e 4, realizou-se uma regressão linear para cada circuito, a qual é utilizada para corrigir automaticamente a potência desejada caso a porcentagem de sólidos do moinho seja diferente de $76 \%$.

\section{RESULTADOS E DISCUSSÃO}

Durante a etapa A, era necessária a presença de um operador para executar a reposição de bolas. O sistema de reposição apresentava problemas mecânicos e a instrumentação existente não era suficiente para detecta-los. A reposição era realizada em grandes bateladas as quais causavam maiores perturbações no processo. Como o cálculo para reposição utilizava apenas um consumo específico fixo e a potência consumida, o grau de enchimento real poderia variar e era apenas corrigido a cada 3 meses durante a medição realizada na parada do circuito.

$\mathrm{Na}$ etapa $\mathrm{B}$, a reposição dispensou a presença do operador e aumentou a frequência de reposição, diminuindo a massa das bateladas e reduzindo a perturbação causada no processo. Entretanto, como a taxa de reposição era fixa, o grau de enchimento poderia variar sendo corrigido apenas a cada 3 meses durante a medição realizada na parada. Nas Figuras 5 e 6 é possível observar no período referente à etapa $\mathrm{B}$ a diminuição da potência com o decorrer do tempo. Ao final da etapa $B$, foi constatada durante a parada, a redução do grau de enchimento, sendo calculada e realizada manualmente uma reposição adicional para correção do grau de enchimento antes do início da etapa C. 


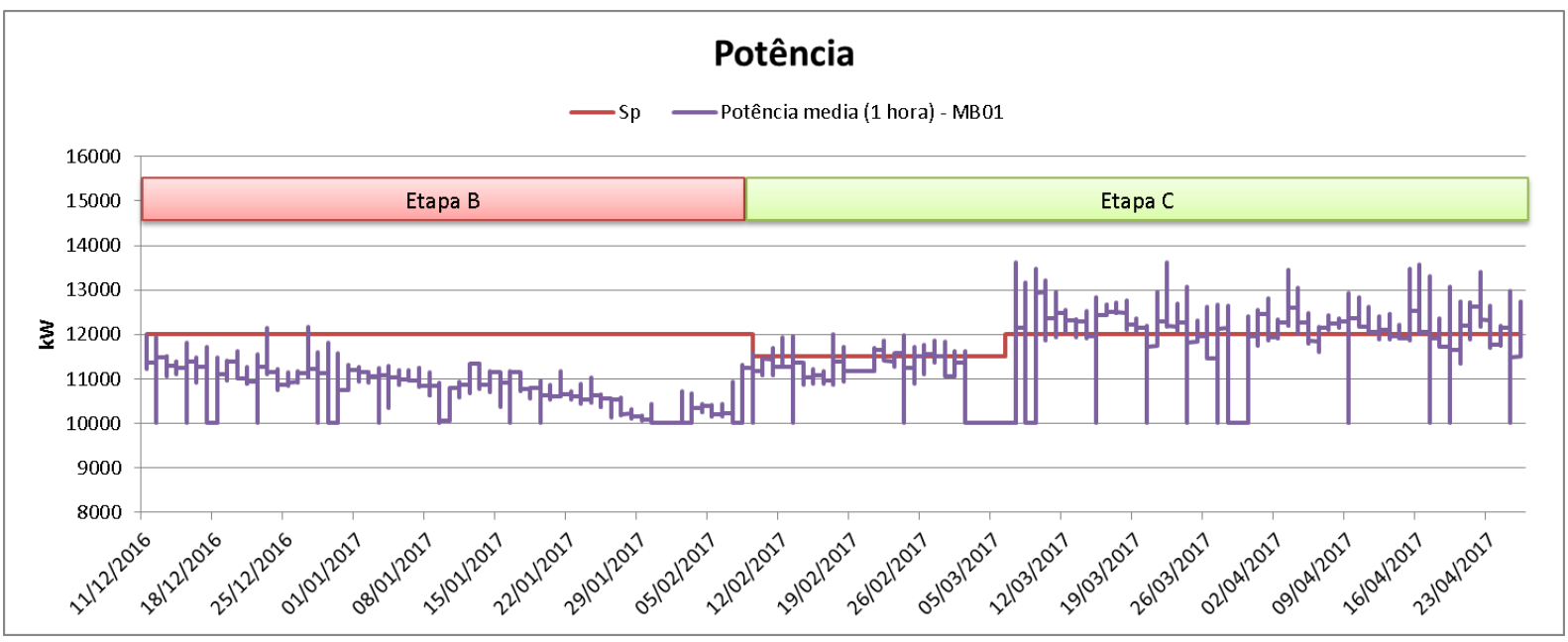

Figura 5. Potência média durante o período das etapas B e C (circuito 1).

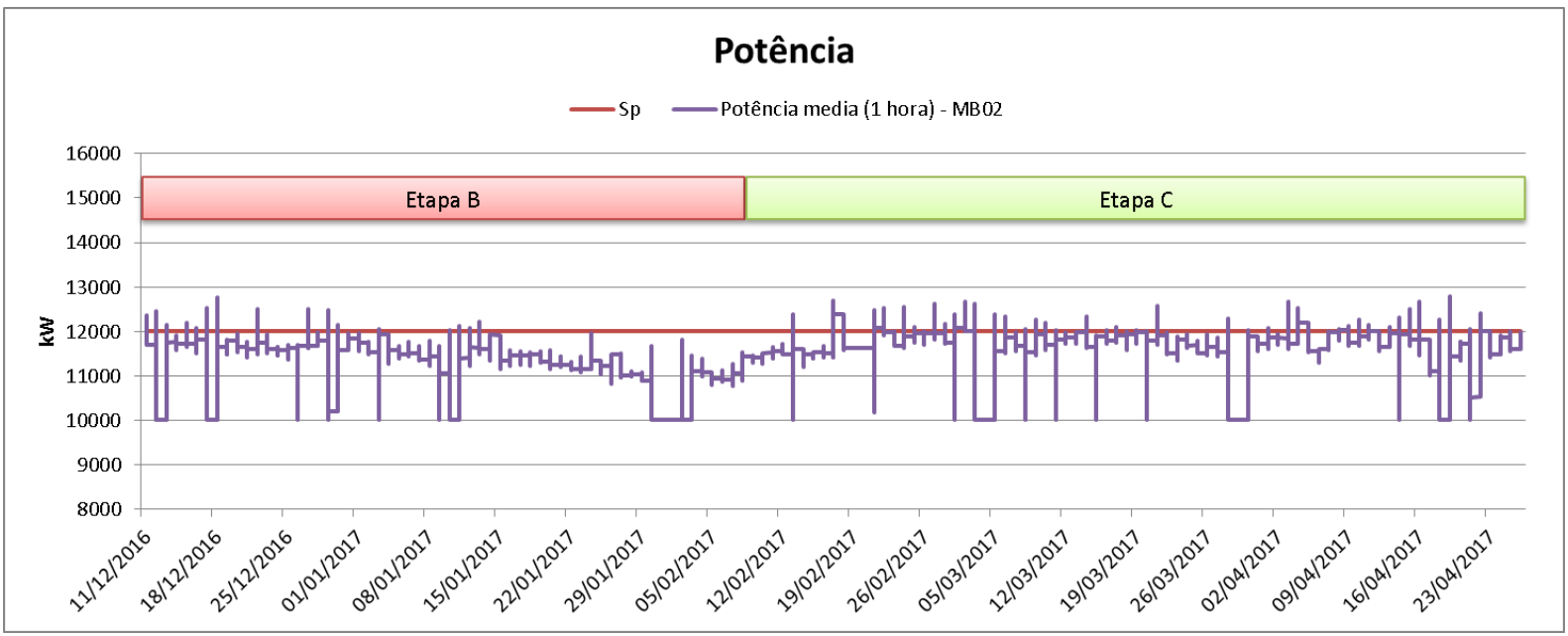

Figura 6. Potência média durante o período das etapas $\mathrm{B}$ e $\mathrm{C}$ (circuito 2).

$\mathrm{Na}$ etapa $\mathrm{C}$, com a variação da taxa de reposição de acordo com a potência, percebeu-se uma maior estabilização da potência, indicando a manutenção do grau de enchimento ao longo do tempo. O setpoint de potência do circuito 1 foi alterado no início de março conforme ilustrado na Figura 5 e razão da troca do revestimento.

A etapa $D$ iniciou junho e será necessário aguardar 3 meses para avaliar os efeitos da nova lógica com a compensação da porcentagem de sólidos do moinho.

\section{CONCLUSÃo}

O sistema de dosagem automático de bolas possibilita uma reposição mais frequente e com menores bateladas, reduzindo perturbações no processo. Além disso, também dispensa a necessidade de acompanhamento por um operador.

O software de controle avançado OCS-4DC foi configurado para alterar a taxa de reposição e manter o grau de enchimento, mesmo que o consumo específico das bolas varie ao longo do tempo. A potência do moinho foi utilizada como indicação indireta do grau de enchimento. Na última etapa do trabalho adicionou-se uma correção para compensar variações na potência causadas por mudanças da porcentagem de sólidos do moinho. 


\section{REFERÊNCIAS}

1 Mazzinghy, D.B., Turrer, H.G.D., Russo, J.F.C., Schneider, C.L., Ramp up da maior instalação de moinhos Vertimill do mundo, XXVI Encontro Nacional de Tratamento de Minérios e Metalurgia Extrativa. 2015; 319-326.

2 Mazzinghy, D.B., Russo, J.F.C., Pimentel, D. A., Parâmetros cinéticos de moagem de itabiritos compacto e friável, HOLOS, Edição Especial - XXV ENTMME / VII MSHNT. 2014;3(30).

3 Gomes, M. P. D.; Santos Júnior, L. T. Controle por imagem permite aumento da alimentação da planta. 10ำ Prêmio de Excelência - Britagem, p.30-32, 2008.

4 Moreira J. L.; Sfalsin L. A.; Júnior Pereira C. P.; Júnior Ataide A. M.; Silva J. M. Melhoria no desempenho do processo de moagem de carvão pela utilização de sistemas otimizantes com lógica nebulosa. $19^{\circ}$ Seminário de Automação e TI Industrial - ABM Week, Rio de Janeiro, 2015.

5 Coutinho S.; Guimarães F.; Totou A.; Bergerman M.; Barbosa R.; Miranda R. C.; Gontijo M. M. Optmizing Control System(c) for desliming and flotation in an iron ore column flotation at the Pico mine. 2nd International Symposium on Iron Ore, São Luís, 2008.

6 Nogueira A.; Pires F. A.; Silva F. T.; Gontijo M. M.; Aplicação de sistema especialista para controle da flotação convencional do concentrador 2 da Samarco. 45 Seminário de Redução de Minério de Ferro e Matérias-primas, 16 Simpósio Brasileiro de Minério de Ferro e $3^{\circ}$ Simpósio Brasileiro de Aglomeração de Minério de Ferro - ABM Week, Rio de Janeiro, 2015.

7 Hogg, R.; Fuerstenau, D. W. Power Relations for Tumbling Mills. Trans. SME-AIME. $1972 ; 252: 418-432$.

8 Sepúlveda, J. E. Methodologies for the Evaluation of Grinding Media Consumption Rates at Full Plant Scale. Minerals Engineering. 2004; 17:1269-1279. 